\title{
Guide d'identification des différentes espèces ou variétés de Fusarium rencontrées en France sur la pomme de terre et dans son environne- ment
}

Bernard TIVOLI

I.N.R.A., Station de Phytopathologie, Centre de Recherches de Rennes, B.P. 29, F 35650 Le Rheu

Il est fréquent d'isoler sur pomme de terre, des champignons du genre Fusarium dont l'identification n'est pas toujours aisée. Les Fusarium isolés à partir de la pomme de terre sont nombreux : on en rapporte au moins 7 espèces ou variétés.

Le guide d'identification des différentes espèces ou variétés de Fusarium rencontrées en France sur la pomme de terre et dans son environnement repose sur un système déductif à trois niveaux croissants de complexité, depuis l'observation morphologique des colonies sur les milieux d'isolement jusqu'à l'observation microscopique permettant une description précise des spores.

Cette identification est réalisée soit directement à partir du milieu d'isolement (milieu gélosé à base d'extrait de malt), soit après repiquage des espèces ou variétés à déterminer sur des milieux spécifiques (milieux PDA et TANAKA).

$\mathrm{Si}$, pour certaines espèces ou variétés de Fusarium $(F$. oxysporum, $F$. solani var. coeruleum, $F$. roseum var. sambucinum), la simple observation des colonies sur le milieu d'isolement permet d'arriver à une identification (niveau I de la détermination), pour d'autres, il sera nécessaire de réaliser les observations après un repiquage sur les milieux spécifiques.

Dans ce cas, l'identification pourra avoir lieu soit par observation de la morphologie des colonies sur ces milieux (niveau II de la détermination lors duquel $F$. roseum var. arthrosporioides est identifié), soit par observation microscopique des spores (niveau III de la détermination; c'est le cas des 3 variétés de F. roseum : graminearum, culmorum et type $\mathrm{X}$ ).

Il est proposé dans ce guide, une description complète des caractéristiques des différentes espèces ou variétés de Fusarium rencontrées sur pomme de terre, ainsi qu'une discussion sur certaines d'entre elles (notamment $F$. roseum var. arthrosporioides et $F$. roseum type $\mathrm{X}$ pour lequel il est proposé $F$. roseum var. gibbosum forme compactum).

Dans la conclusion, il est fait état de l'évolution de la flore fusarienne et de sa dynamique durant la culture et la conservation de la pomme de terre.

Mots clés additionnels : Systématique, caractéristiques, dynamique, population. occurring on potato and in its immediate environment in France.

Fungi of the genus Fusarium are commonly isolated from potato plants and tubers. Their identification is not always easy and at least 7 species or varieties have been recorded from potatoes. This guide to the identification of the species and varieties of Fusarium which have been found in France on the potato plant, both from the tuber and its immediate soil or store environment, depends on an identification system with three levels of complexity. These range from observations on colony morphology on the isolation medium, to a precise description of the spores under the microscope. Identification can be carried out either directly on the isolation medium (e.g. malt extract agar) or after transfer to a more specific medium (c.g. PDA, TANAKA). Although for certain species and varieties (Fusarium oxysporum, $F$. solani var. coeruleum, $F$. roseum var. sambucinum) simply observing the culture on its isolation medium will allow it to be identified (first level of identification), for others it is necessary to study it after transfer to a specific medium. In this case, the identification can be made either by observing the morphology of the colony on the medium (second level of identification, by which $F$. roseum var. arthrosporioides can be recognized) or by looking at 
the spores under the microscope (third level of identification, appropriate for three varieties of $F$. roseum : graminearum, culmorum and type X).

The guide gives a complete description of the characteristics of the different species and varieties of Fusarium from the potato, and the taxonomy of some of them is discussed (particulary $F$. roseum var. arthrosporioides and $F$. roseum type $\mathrm{X}$; for the latter, the name $F$. roseum var. gibbosum forme compactum is proposed). Conclusions are made concerning the development of the fungal flora of the Fusarium group and its population dynamics during the growth and storage of the potato.

Additional key words : Classification, characteristics, dynamics, population.

\section{INTRODUCTION}

La pomme de terre est accompagnée, aussi bien lors de sa phase de végétation que lors de la conservation du plant, de tout un cortège fongique contribuant, à des degrés divers, à diminuer sa production et (ou) sa qualité.

Dans la recherche des parasites, il est fréquent d'isoler des champignons appartenant au genre Fusarium dont l'identification n'est pas toujours aisée.

\section{A. Les objectifs de ce guide}

On peut s'étonner de voir paraître un guide de détermination des Fusarium dans le cas précis de la pomme de terre dans la mesure où, déjà, plusieurs ouvrages traitant de l'ensemble des Fusarium sont proposés au phytopathologiste : BOOTH (1977), TOUSSOUN \& NELSON (1976) et, plus récemment NELSON et al. (1983). Ce dernier fascicule offre l'avantage de mentionner la synonymie complète entre les sections, espèces et variétés de Fusarium ainsi qu'une iconographie en couleur fort utile. les :

Le présent guide se justifie par 2 raisons principa-

- Les Fusarium isolés à partir de la pomme de terre en France sont nombreux : on en rapporte au moins 7 espèces ou variétés. Un recueil adaptant, pour une espèce végétale, les méthodes à suivre pour arriver à une identification et regroupant les différentes espèces ou variétés de Fusarium susceptibles d'être rencontrées est particulièrement précieux pour la personne chargée de la détection de ces champignons.

- Il n'existe pas de description complète des différentes souches isolées de pomme de terre et certaines possèdent, semble-t-il, des caractéristiques particulières qui les distinguent des formes décrites jusqu'à présent.

\section{B. Les Fusarium et leur classification}

Il n'est pas utile, dans le cadre de cet article de revenir sur les nombreuses descriptions des Fusarium qu'elles soient basées sur la clé de détermination de WOLLENWEBER \& REINKING (1935) avec ses $16 \mathrm{sec}-$ tions et 50 espèces, reprise par BOOTH (1971) et GERLACH \& NIRENBERG (1982) ou sur celle de SNYDER \& HANSEN (MESSIAEN, 1959) reprise par MESSIAEN \& CASSINI (1968) et réduite à 10 espèces. Ces derniers auteurs adoptent les grandes lignes de la classification de SNYDER \& HANSEN car d'après eux, à l'intérieur des 10 espèces décrites, "les variations morphologiques, souvent importantes n'ont aucune valeur taxonomique puisque par mutation ou recombinaison sexuelle, les diverses formes peuvent expérimentalement dériver les unes des autres ". Certaines de ces espèces sont subdivisées en variétés ou (et) en formes spéciales.

Sans rentrer dans le détail de la taxonomie, il est néanmoins indispensable d'être précis et de reclasser les espèces ou variétés de Fusarium rencontrées sur pomme de terre dans le contexte de l'ensemble des Fusarium. La classification choisie est celle de MESSIAEN \& CASSINI (1968) mais nous ferons parfois référence aux autres classifications afin d'éviter les confusions et faciliter la compréhension de certains articles basés sur l'une ou l'autre des clés de détermination.

Nous nous limiterons aux seules espèces ou variétés de Fusarium isolées le plus fréquemment en France à partir des différents organes de la pomme de terre (tubercules, tiges, stolons, racines) ou de son environnement (sol et locaux de conservation). Il sera parfois fait référence à quelques espèces ou variétés autres avec lesquelles il existe un risque de confusion.

\section{Les étapes de la détermination}

La détermination des Fusarium de la pomme de terre se réalise en 2 étapes successives parfois complémentaires :

- La $1^{\text {re }}$ consiste en une identification directe sur le milieu d'isolement de quelques espèces ou variétés de Fusarium à partir des caractéristiques culturales : pigmentation, aspect du mycélium et fructification. Ce sera le cas pour $F$. oxysporum, $F$. solani var, coeruleum et $F$. roseum var. sambucinum.

- La $2^{\mathrm{e}}$ consiste à repiquer sur 2 milieux spécifiques, les isolats non identifiés précédemment. C'est le cas essentiellement des $F$. roseum caractérisés par leur pigmentation rouge carmin et souvent un mycélium aérien abondant. Ces milieux induisent une pigmentation particulière et l'apparition plus ou moins abondante de tel ou tel type de spores dont la description lors de l'observation microscopique, conduit à l'identification de la variété de $F$. roseum.

Ces repiquages permettent, en outre, d'éliminer la plupart des champignons autres que les Fusarium qui peuvent être associés aux colonies, en particulier ceux qui possèdent des spores uni ou bicellulaires évoquant 
des microconidies (Verticillium, Cephalosporium, Acrostalagmus ou Spicaria : MESSIAEN \& CASSINI, 1968).

Cette synthèse est subdivisée en 4 parties :

- Le matériel et les méthodes d'étude.

- La clé de détermination.

- Identification directe sur milieux d'isolement : $F$. oxysporum, F. solani var. coeruleum, F. roseum var. sambucinum.

- Identification après repiquage sur les milieux spécifiques: $F$. roseum var. arthrosporioides, $F$. roseum var. culmorum, $F$. roseum var. graminearum, F. roseum type X.

Enfin, dans la conclusion, il sera fait état de la distribution écologique des différentes espèces ou variétés de Fusarium rencontrées en France, sur la pomme de terre ou dans son environnement.

\section{LE MATÉRIEL ET LES MÉTHODES D'ÉTUDES}

\section{A. Les milieux de culture}

Afin de limiter au mieux le développement des bactéries, l'addition de streptomycine aux milieux de culture après autoclavage à une dose de $250 \mathrm{ppm}$ est recommandée.

\section{Le milieu d'isolement}

Les isolements fongiques sont généralement réalisés sur un milieu gélosé à base d'extrait de malt à 2 p. 100 .

\section{Les milieux spécifiques}

Au nombre de 2, ils permettent une identification précise des différentes variétés de Fusarium roseum autres que $F$. roseum var. sambucinum :

- milieu PDA à $200 \mathrm{~g}$ d'extrait de pomme de terre ;

- milieu TANAKA (OU, 1972, modifié selon BOISSONNET-MENES \& LECOQ, 1976) dont la composition par litre est la suivante :

Glucose : 20 g- $\mathrm{KNO}_{3}: 3 \mathrm{~g}-\mathrm{PO}_{4} \mathrm{KH}_{2}: 1 \mathrm{~g}-\mathrm{PO}_{4} \mathrm{~K}_{2} \mathrm{H}$ : $1 \mathrm{~g}-\mathrm{MgSO}_{4}, \quad 7 \mathrm{H}_{2} \mathrm{O}: 0,5 \mathrm{~g}-\mathrm{CaCl}_{2}, 2 \mathrm{H}_{2} \mathrm{O}: 0,1 \mathrm{~g}-$ $\mathrm{FeSO}_{4}, 7 \mathrm{H}_{2} \mathrm{O}: 7,5 \mathrm{mg}-\mathrm{MnSO}_{4}, 7 \mathrm{H}_{2} \mathrm{O}: 2 \mathrm{mg}-\mathrm{CuSO}_{4}$, $5 \mathrm{H}_{2} \mathrm{O}: 6 \mathrm{mg}-\mathrm{ZnCl}_{2}: 75 \mathrm{mg}-\left(\mathrm{NH}_{4}\right)_{6} \mathrm{Mo}_{7} \mathrm{O}_{24}: 9 \mathrm{mg}-$ Biotine : $5 \mu \mathrm{g}$-Thiamine : $1 \mathrm{mg}$-Gélose : $20 \mathrm{~g}$.

Le repiquage des Fusarium à identifier s'effectue systématiquement sur les 2 milieux à la suite du prélèvement des explants fongiques en bordure des colonies développées sur les milieux d'isolement, le plus loin possible des fragments végétaux.

\section{B. Les méthodes d'isolement et les conditions de culture}

Les isolements fongiques se réalisent en boîtes de Petri, comme pour de nombreux autres champignons, par dépôt sur milieu de culture de fragments végétaux préalablement désinfectés par trempage dans l'alcool à $95^{\circ}$ pendant 30 à $45 \mathrm{~s}$ puis rincés abondamment à l'eau stérile (au moins 3 rinçages successifs) et séchés. L'apport d'antibiotique dans le milieu, le dépôt des fragments végétaux sur un milieu sec ainsi qu'un séchage minutieux des fragments végétaux permet d'éviter la prolifération de colonies bactériennes.

Les boîtes d'isolement demeurent à incuber au laboratoire dans les conditions de lumière naturelle à une température ambiante voisine de $20^{\circ} \mathrm{C}$.

Par contre, les boîtes de repiquage sont soumises à un éclairement de 10000 lux avec une photopériode de $12 \mathrm{~h}$, à une température de $25^{\circ} \mathrm{C}$. Ces conditions de culture préconisées par MESSIAEN \& CASSINI (1968) sont indispensables à l'expression des caractères recherchés.

\section{Les identifications}

L'identification repose sur une observation globale intégrant à la fois la morphologie des colonies et l'observation microscopique des spores; elle s'effectue après $8 \mathrm{j}$ de culture.

Les colonies, développées à la suite de la croissance plus ou moins rapide du mycélium (appréciée à $25^{\circ} \mathrm{C}$ ), sont caractérisées par un mycélium pigmenté ou non, aérien ou ras. L'observation de la pigmentation est réalisée au niveau du mycélium aérien et sur le mycélium situé au contact direct de la gélose (cette dernière observation s'effectue en retournant la boîte). De plus, les spores parfois groupées en sporodochies (fructifications dans laquelle la masse conidienne est supportée par un stroma couvert de courts conidiophores), peuvent donner à la colonie un aspect « gras».

L'observation microscopique prend en compte la forme et la taille des macroconidies, la présence ou l'absence de microconidies et de chlamydospores. Dans le cas où ces derniers types d'organes sont observés, la description de leur forme et de leur taille s'impose.

\section{CLÉ DE DÉTERMINATION}

La clé de détermination proposée (tabl. 1), a davantage pour but de répondre à un objectif pratique que de dresser un tableau exhaustif des caractères systématiques des différentes espèces ou variétés de Fusarium rencontrées. Elle repose donc sur un système déductif (à 3 niveaux de complexité) depuis l'observation morphologique des colonies sur les milieux d'isolements jusqu'à l'observation microscopique permettant une description précise des spores.

$\mathrm{Si}$, pour certaines espèces ou variétés de Fusarium (F. oxysporum, F. solani var. coeruleum, F. roseum var. sambucinum), la simple observation des colonies sur le milieu d'isolement permet d'arriver à une identification (niveau 1 de la détermination), pour d'autres, il sera nécessaire de réaliser les observations après un repiquage sur les milieux spécifiques.

Dans ce cas, l'identification pourra avoir lieu soit par observation de la morphologie des colonies sur ces milieux (niveau 2 de la détermination, c'est le cas de F. roseum var. arthrosporioides), soit par observation microscopique des spores (niveau 3 de la détermination, c'est le cas des variétés de $F$. roseum, graminearum, culmorum et type X).

L'identification basée sur l'observation de la mor- 

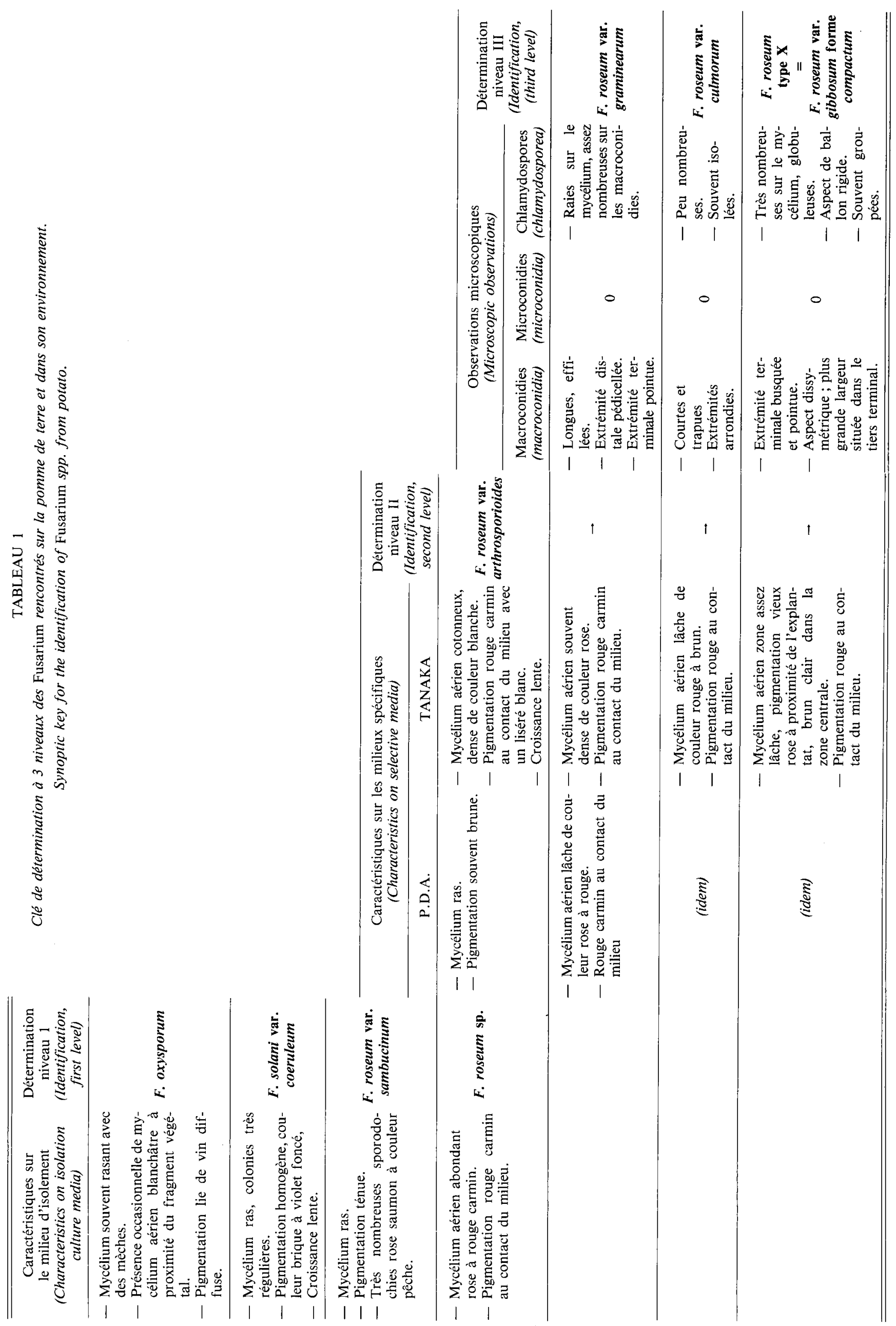
phologie des colonies (niveaux I et II), n'exclut évidemment pas de conforter sa détermination par l'observation des spores ; c'est la raison pour laquelle celles-ci sont décrites ultérieurement pour chacune des espèces ou variétés de Fusarium considérées.

\section{IDENTIFICATION DIRECTE SUR LES MILIEUX D'ISOLEMENT}

A. Fusarium oxysporum Schlecht. emend Sn. \& H. (fig. 1, 2, 3)

Ce champignon est fréquemment isolé à partir de différents organes de la plante en végétation (tiges, racines, stolons), et beaucoup plus rarement à partir des tubercules en conservation.

Les colonies, généralement caractérisées par un mycélium aérien blanc grisâtre assez lâche, ont l'aspect d'un cône aplati en raison d'un mycélium beaucoup plus développé dans la partie proche du fragment végétal qu'au niveau de la zone frontale de la colonie. Le pourtour de la culture est souvent caractérisé par un mycélium ras en forme de mèches.

La pigmentation du mycélium au contact du milieu de culture est, la plupart du temps, blanchâtre pendant les 15 premiers jours qui suivent l'isolement, puis se pigmente, souvent de façon diffuse, dans la zone centrale, d'une couleur lie de vin.

$F$. oxysporum est caractérisé par la présence abondante de microconidies ellipsoïdales $(4,5-5,5 \mu \mathrm{m} \times 1,5$ $2,5 \mu \mathrm{m})$ et de macroconidies de taille variable mais n'excédant généralement pas $20 \mu \mathrm{m}$ de longueur $(13-20 \mu \mathrm{m} \times 2-3 \mu \mathrm{m})$. Ces spores sont regroupées sous forme de fausses têtes sèches à l'extrémité de microconidiophores allongés, dispersés sur le mycélium aérien. Après 3 semaines de culture, de nombreuses chlamydospores intercalaires apparaissent sur le mycélium.

Il arrive que ce champignon soit confondu sur milieu d'isolement avec des $F$. roseum peu pigmentés ce qui rend parfois nécessaire son repiquage sur les milieux PDA et TANAKA. Sur le premier milieu, les caractéristiques décrites précédemment sont plus accusées, notamment en ce qui concerne sa pigmentation au contact du milieu de culture; de plus, le mycélium est souvent ras. Sur le milieu TANAKA, la colonie est caractérisée par un mycélium aérien très abondant et extrêmement dense, de couleur blanche immaculée, aussi bien en surface qu'au contact du milieu. Toutes les souches isolées à partir de la pomme de terre appartiennent au type "cotonneux " décrit par MESSIAEN \& CASSINI (1968). La croissance de $F$. oxysporum sur ces milieux de culture, à $25^{\circ} \mathrm{C}$ est comprise entre 7 et $7,4 \mathrm{~mm}$ par jour.

\section{B. Fusarium solani var. coeruleum (Sacc.) Booth.} (fig. 1, 2, 3)

Ce champignon est fréquemment détecté dans le sol situé à proximité des parties souterraines des plantes ainsi que sur les tubercules en voie de pourriture.

Il est caractérisé sur milieu d'isolement par un mycélium ras, un aspect des colonies souvent poudreux et une pigmentation homogène, même sur de jeunes colonies, de couleur brique, parfois violet foncé. En mélange avec d'autres champignons, il peut parfois prendre une coloration lie de vin qui évoque celle de $F$. oxysporum. Mais sa croissance lente, le pourtour régulier des colonies bordées d'un mycélium très fin et l'observation des spores au microscope permettent de rectifier une éventuelle erreur d'identification.

Comme $F$. oxysporum, il possède des microconidies mais celles-ci sont ovoïdes, regroupées dans une gouttelette au bout d'un microconidiophore allongé, peu nombreuses et plus massives $(5-6 \mu \mathrm{m} \times 2,5-3 \mu \mathrm{m})$; de plus, ses macroconidies sont allongées, assez peu incurvées avec des extrémités très arrondies (30$35 \mu \mathrm{m} \times 2,5-3 \mu \mathrm{m})$. Sur des colonies âgées de 3 semaines et plus, ce champignon forme des chlamydospores abondantes aussi bien sur le mycélium que sur les macroconidies. Elles sont arrondies, en ballonnet, souvent en chaînette sur le mycélium, avec des doubles parois très caractéristiques.

Après repiquage sur PDA, à $25^{\circ} \mathrm{C}$ la colonie possède les mêmes caractéristiques morphologiques que précédemment ; sur le milieu TANAKA, le mycélium aérien abondant et dense, au début de couleur blanche, prend progressivement, soit sous forme de zonations, soit de façon homogène, une couleur bleue azurée très caractéristique. Sa vitesse de croissance sur ces 2 milieux est la plus faible de tous les Fusarium de la pomme de terre puisqu'elle varie entre 3 et $3,8 \mathrm{~mm}$ par jour.

\section{Fusarium roseum var. sambucinum (Fuck.) Sn. \& H.}

$$
\text { (fig. 1, 2, 3) }
$$

Ce champignon est très fréquemment isolé à partir des tubercules en voie de pourriture et également à partir des tiges après le défanage. Il fait l'objet d'une synonymie importante: $F$. sambucinum Fuckel., $F$. sulphureum Schlecht. ou encore $F$. sambucinum F. 6.

Facile à déterminer sur milieu d'isolement, il est caractérisé par un mycélium blanc, généralement ras, pouvant parfois, chez certaines souches devenir aérien très lâche. Le long du mycélium situé au contact de la gélose, se développent, dès le $4^{\mathrm{e}} \mathrm{j}$ après l'isolement des sporodochies de couleur rose saumon, très rapprochées les unes des autres, qui donnent à la colonie un aspect gras et une couleur d'ensemble rosée.

$\mathrm{Ce}$ champignon possède uniquement des macroconidies courtes, caractérisées par des extrémités anguleuses, brutalement pointues (18-22 $\mu \mathrm{m} \times 2,5-3 \mu \mathrm{m})$.

Sur les milieux PDA et TANAKA, il possède sensiblement la même morphologie que celle précédemment décrite avec une vitesse de croissance variant entre 7 et 7,5 mm par jour.

Sur le milieu d'isolement il pourrait être confondu avec $F$. lateritium (Ness) Sn. \& H. caractérisé par des sporodochies regroupées en pionnotes qui recouvrent la quasi-totalité de la zone située à proximité du fragment végétal (sur $1 \mathrm{~cm}^{2}$ ). Ce champignon d'une part, n'a jamais été signalé sur pomme de terre et, d'autre part, se développe beaucoup moins rapidement en culture pure et possède des macroconidies très particulières, rétrécies à l'extrémité. MESSIAEN \& CASSINI 

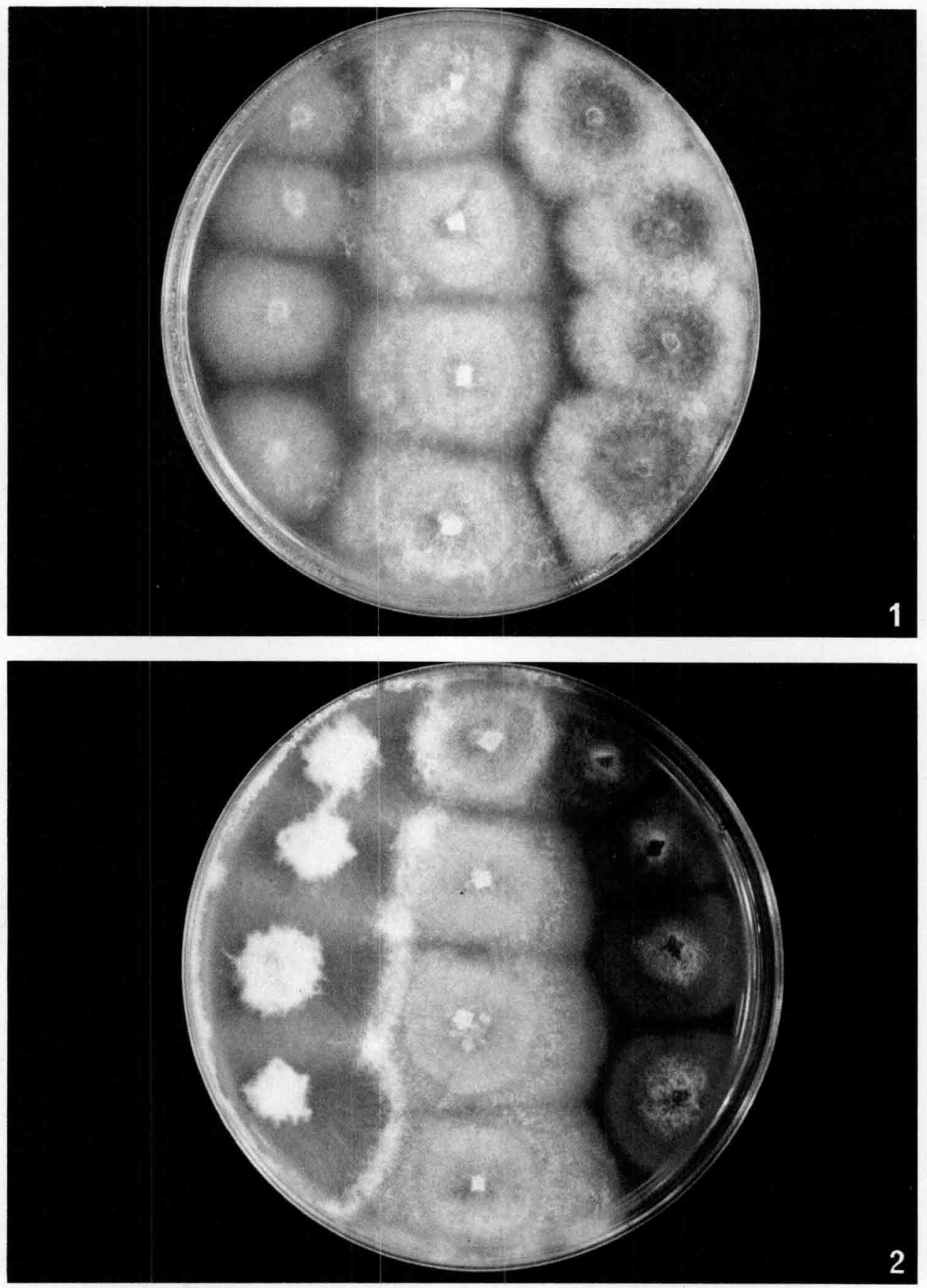

Figure 1

Morphologie de F. roseum var. sambucinum, F. solani var. coeruleum et des autres F. roseum sur milieux d'isolement.

Morphology of $\mathrm{F}$. roseum var. sambucinum, F. solani var. coeruleum, F. oxysporum and others F. roseum on isolation medium).
1. De gauche à droite (left to right): F. solani var. coeruleum, $\mathrm{F}$. roseum var. sambucinum et $\mathrm{F}$. roseum.

2. De gauche à droite (left to right) : F. oxysporum, F. roseum var sambucinum et $\mathrm{F}$. solani var. coeruleum. 


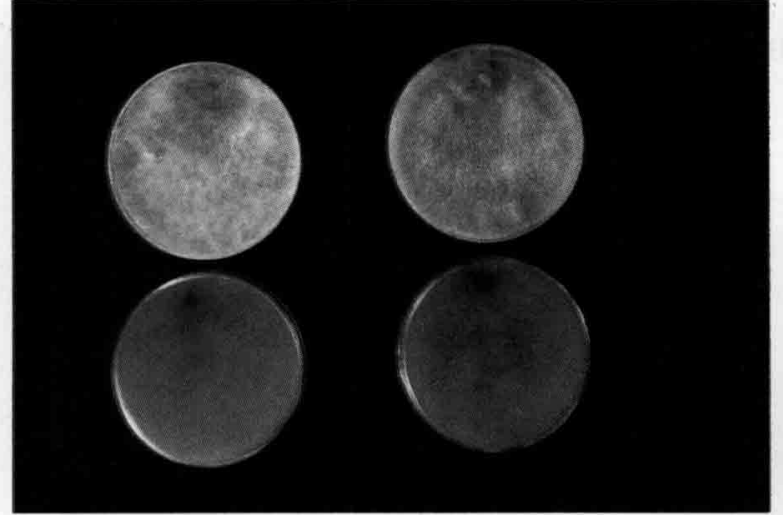

F. roseum var. culmorum

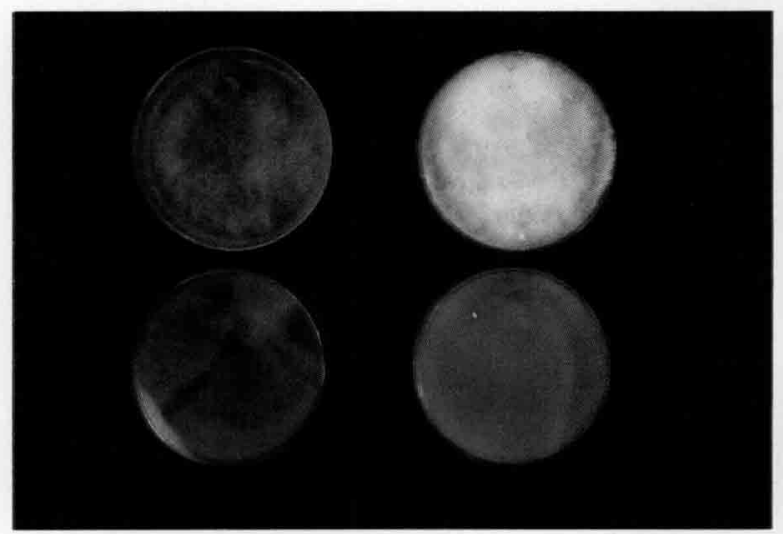

F. roseum var. graminearum

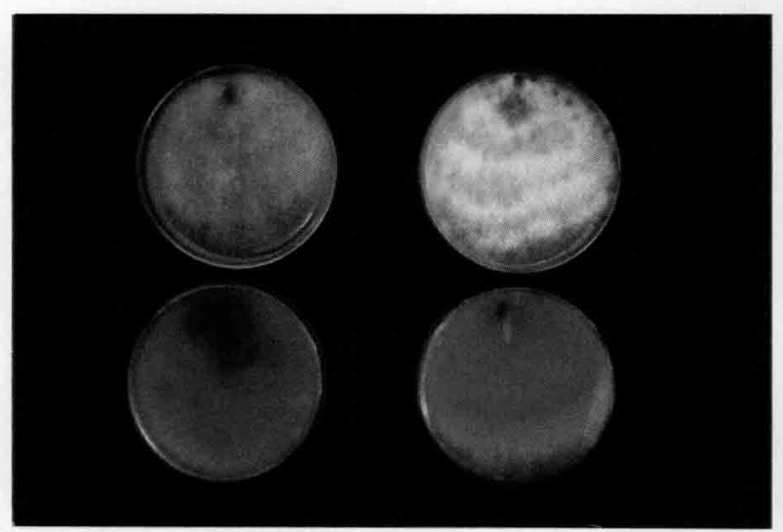

F. roseum var. gibbosum f. compactum

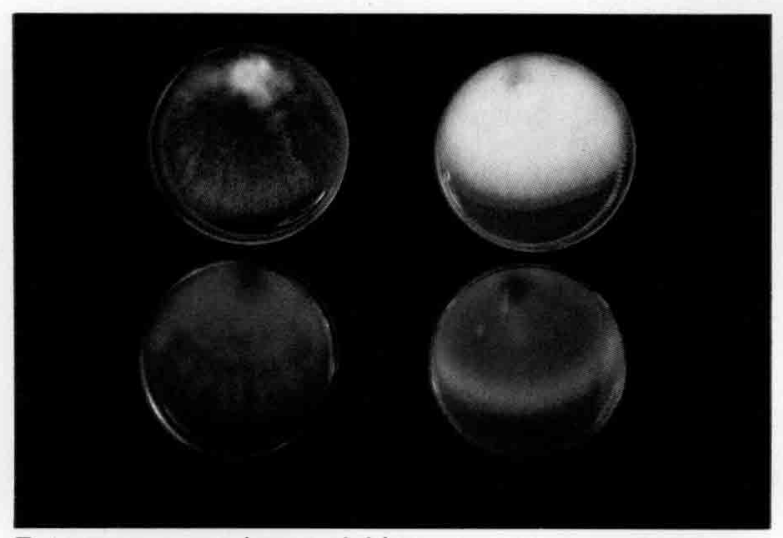

F. roseum var. arthrosporioides

Figure 2

Aspects des colonies sur les mulieux P.D.A. (à gauche) et TANAKA (à droite), observées faces supérieures (en haut) et inférieures (en bas).

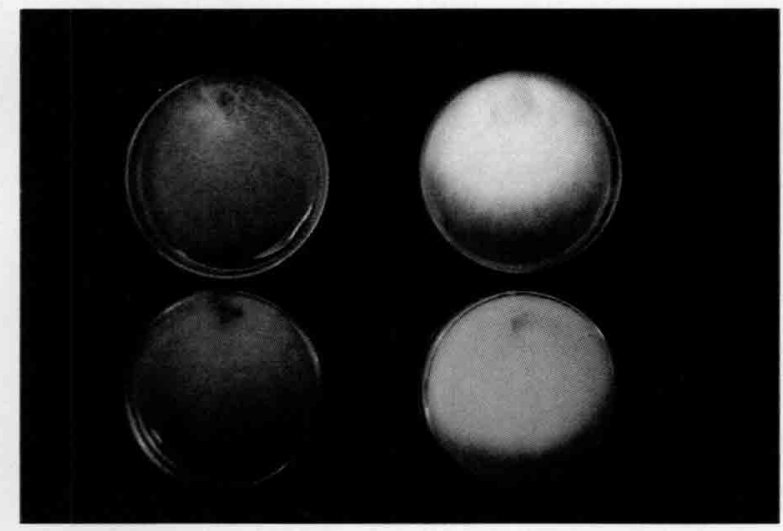

\section{F. oxysporum}

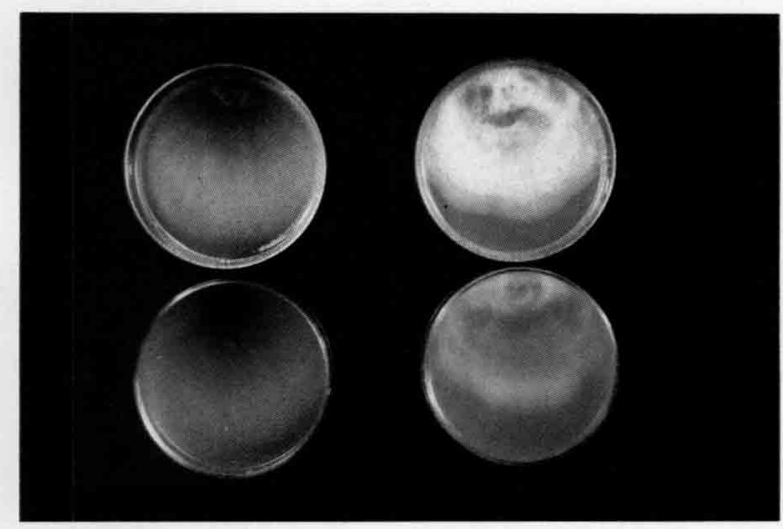

F. solani var. coeruleum

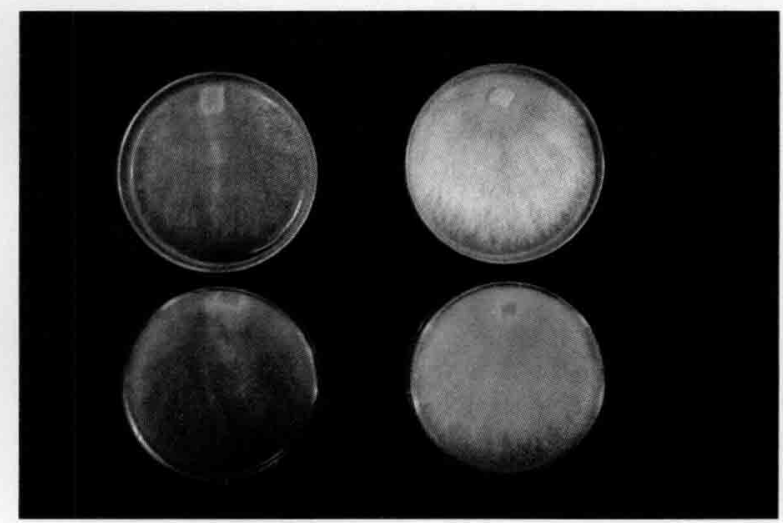

F. roseum var. sambucinum
Morphology on media P.D.A. (on the left) and Tanaka (on the right), upper (top) lower (bottom). 

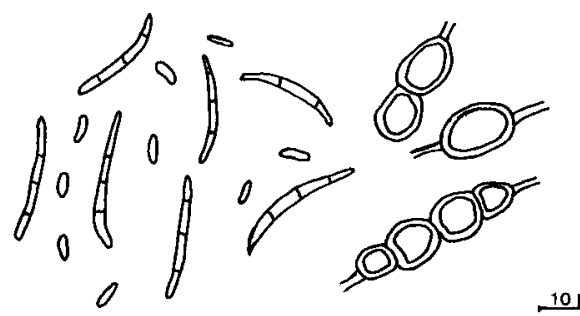

$\stackrel{10 \mu .}{\longrightarrow}$

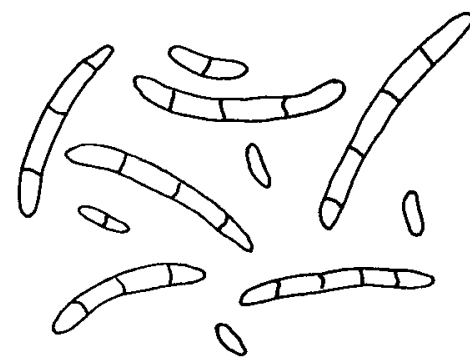

F. solani var. coeruleum

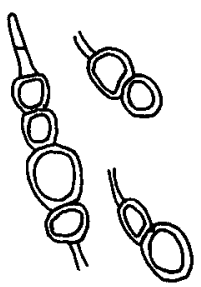

$\stackrel{10 \mu}{\longrightarrow}$

F. oxysporum

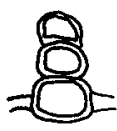

Q0)
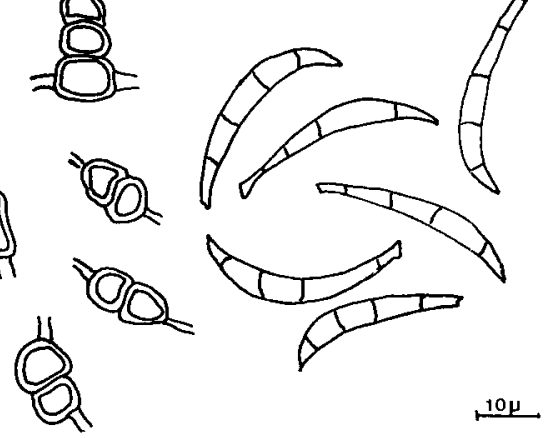

$F$. roseum var. gibbosum $F$. compactum

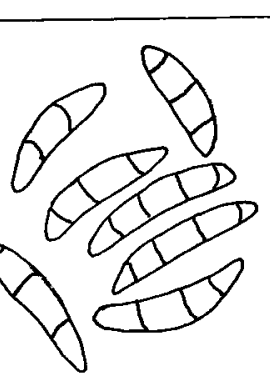

$\stackrel{10 \mu}{\longrightarrow}$

Type 1

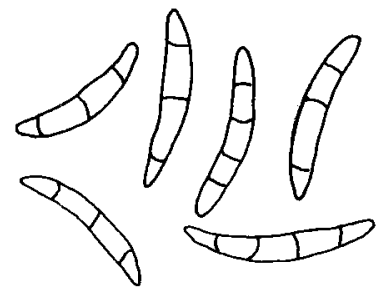

10ب

Type 2

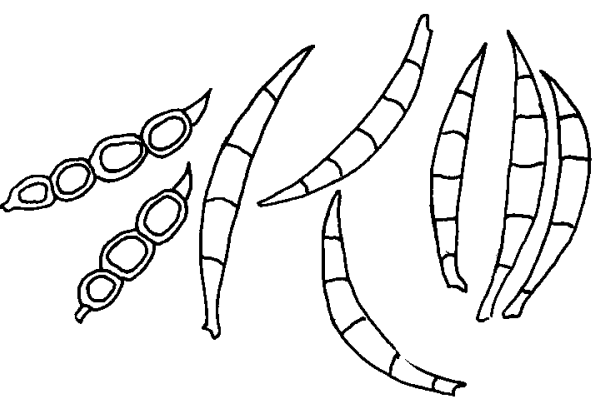

104

F. roseum var. graminearum

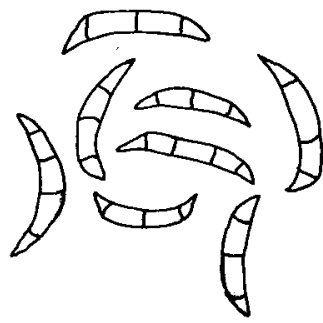

104

F. roseum var. sambucinum

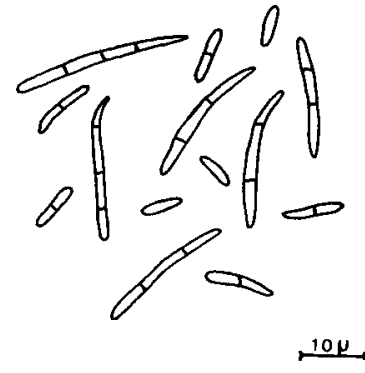

type « arthrosporioides »

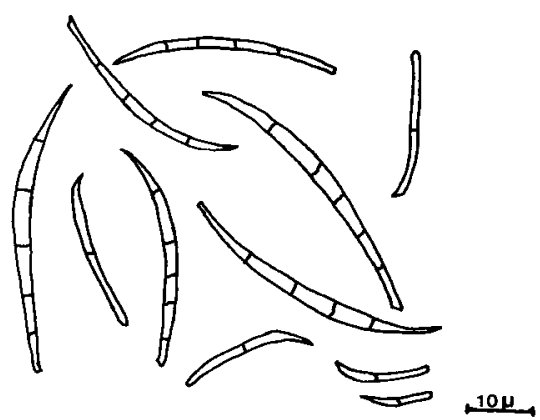

type « avenaceum "

F. roseum var. arthrosporioides

Figure 3

Caractéristiques des spores des différentes espèces ou variétés de Fusarium rencontrées sur pomme de terre ou dans son environnement. Characteristics of the spores of different species and varieties of Fusarium found on potatoes or in their environment. 
(1968) soulignent toutefois une certaine parenté entre ces deux espèces.

\section{IDENTIFICATION APRÈS REPIQUAGE SUR LES MILIEUX SPÉCIFIQUES}

Caractérisés par un mycélium aérien le plus souvent abondant allant du blanc, au rose ou au brun et une pigmentation, rouge carmin au contact de la gélose, les $F$. roseum de la pomme de terre mis à part $F$. roseum var. sambucinum appartiennent à 4 variétés différentes.

En raison de leur association fréquente, d'une part avec des bactéries qui modifient souvent leur pigmentation (au jaune ou à l'orange) et, d'autre part, avec d'autres champignons à spores uni ou bicellulaires, leur repiquage, en vue de leur identification, sur les milieux PDA et TANAKA, s'avère indispensable. Sur milieu d'isolement, on peut parfois confondre certaines colonies de $F$. roseum avec celles d'Epicoccum $\mathrm{sp}$.

\section{A. Fusarium roseum var. arthrosporioides (Sherb.) MESSIAEN \& CASSINI (fig. 2 et 3 )}

Ce Fusarium est fréquent sur tubercules en voie de pourriture et est assez rarement isolé à partir de la plante ou du sol.

Sa morphologie sur le milieu TANAKA, différente de celle des autres $F$. roseum, permet une identification rapide : il possède un mycélium aérien blanc, abondant, cotonneux et très dense, rappelant celui de $F$. oxysporum; au contact du milieu, il prend une coloration rouge carmin intense avec une zone blanche de $5 \mathrm{~mm}$, non encore pigmentée, au front de croissance de la colonie (c'est la raison pour laquelle sa détermination est facilitée lorsque la colonie n'a pas encore atteint le bord de la boîte).

Sur le milieu PDA, la colonie est plus irrégulière, le mycélium est ras et sa pigmentation au contact de la gélose est souvent diffuse et brune. La vitesse de croissance de ce Fusarium est la plus faible des $4 \mathrm{~F}$. roseum puisque, à $25^{\circ} \mathrm{C}$, elle varie, selon les souches, de 6,5 à 7,2 mm par jour.

De plus, $F$. roseum var. arthrosporioides est le seul du groupe des $F$. roseum à posséder des microconidies. Celles-ci sont uni ou bicellulaires, ellipsoïdales, ressemblant à celles de $F$. oxysporum mais de longueur très variable $(9-18 \mu \mathrm{m} \times 2,5 \mu \mathrm{m})$. Les macroconidies sont parfois difficiles à distinguer des microconidies les plus allongées car elles sont également très étroites $(2,5 \mu \mathrm{m})$; elles possèdent en général 3 cloisons et leur longueur varie entre 22 et $27 \mu \mathrm{m}$. Les 2 types de spores sont répartis le long du mycélium aérien.

Sur des milieux organiques, non décomposés (pailles ou grains d'orge), mais très rarement sur le milieu TANAKA (à proximité immédiate de l'implant gélosé initial), les macroconidies peuvent être groupées en sporodochies volumineuses (entre 2 et $3 \mathrm{~mm}$ ) possédant une morphologie semblable à celles des macroconidies de $F$. roseum var. avenaceum (Link) Sn. \& $H$. : très allongées, étroites (31-41 $\mu \mathrm{m} \times 2,5-3 \mathrm{~m})$, possédant 5 à 6 cellules. On observe souvent, parmi ces macroconidies typiques, des conidies de forme et de taille variables la plupart du temps bicellulaires
(10-27 $\mu \mathrm{m} \times 1,5-2 \mu \mathrm{m})$. Les colonies issues d'isolats monospores de type "avenaceum » présentent toujours sur les milieux PDA et TANAKA les mêmes caractéristiques que $F$. roseum var. arthrosporioides décrites précédemment. Enfin, l'existence de ces sporodochies volumineuses n'exclut pas, à d'autres endroits du milieu de culture, la présence des 2 types de spores typiques de $F$. roseum var. arthrosporioides.

Il est vraisemblable que dans le cas de la pomme de terre, $F$. roseum var. arthrosporioides et $F$. roseum var. avenaceum mentionnés par de nombreux auteurs ne soient en fait qu'une seule et même variété de $F$. roseum. C'est la conclusion à laquelle nous sommes arrivé à la suite de ces repiquages et d'échanges entre souches avec les Dr TURKENSTEEN (Pays-Bas) et NIRENBERG (R.F.A.). Cette idée est renforcée par les travaux de CORMACK (1951) qui montre que les limites entre $F$. arthrosporioides Sherb. et $F$. avenaceum (Fr.) Sacc. sont loin d'être précises et que les variants de l'un ou l'autre des champignons peuvent conduire à l'autre espèce.

\section{B. Fusarium roseum var. culmorum (Schwabe) Sn. \&} H. (fig. 2 et 3 )

Ce champignon est isolé à partir des tubercules en voie de pourriture et des parties aériennes des plantes.

La morphologie de ses colonies ne permet pas à elle seule de l'identifier : le mycélium aérien est lâche, la pigmentation varie $\mathrm{du}$ rouge carmin au brun sur les 2 milieux de repiquage.

La vitesse de croissance des souches de $F$. roseum var. culmorum à $25^{\circ} \mathrm{C}$ est comprise entre 12,2 et $12,7 \mathrm{~mm}$ par jour.

Ce champignon possède des macroconidies souvent abondantes réparties sur le mycélium en microsporodochies, ainsi que quelques chlamydospores volumineuses, hyalines, sans rigidité apparente, réparties sur les hyphes.

Les macroconidies, à extrémités arrondies, sont caractéristiques de 2 types de souches isolées à partir de la pomme de terre ou de son environnement :

- type 1, caractérisé par des macroconidies possédant généralement 3 à 4 cellules, d'aspect trapu et de petite taille $(19-27 \mu \mathrm{m} \times 4-8 \mu \mathrm{m})$;

- type 2, caractérisé par des macroconidies possédant 4 à 5 cellules, moins massives que les précédentes et plus allongées $(26-31 \mu \mathrm{m} \times 4-6 \mu \mathrm{m})$.

\section{Fusarium roseum var. graminearum (Schwabe)} Sn. \& H. (fig. 2 et 3 )

La distribution de ce champignon est identique à celle de $F$. roseum var. culmorum.

Comme pour ce champignon, aucune caractérisation ne peut être réalisée par simple observation de la morphologie des colonies qui présentent des caractéristiques identiques. On note souvent sur le milieu TANAKA, la présence d'un mycélium aérien compact de couleur rosée.

La vitesse de croissance des souches varie à $25^{\circ} \mathrm{C}$, entre 10,6 et $11 \mathrm{~mm}$ par jour.

Les macroconidies se développent surtout au con- 
tact du milieu gélosé, sous le mycélium aérien et sont peu nombreuses. Elles sont très caractéristiques : de courbure parfaite, elles possèdent une extrémité distale pédicellée, une extrémité terminale pointue et sont constituées de 5 à 6 cellules. De taille assez imposante $(27-40 \mu \mathrm{m})$, leur plus grande largeur $(4-6 \mu \mathrm{m})$ se situe dans leur partie médiane.

Il arrive que l'on observe sur les macroconidies, des chlamydospores en forme de ballonnets intercalaires d'apparence très rigide. Ces souches de $F$. roseum var. graminearum s'apparentent aux souches de type " sol » définies par MESSIAEN et al. (1976), contrairement à celles de type «céréales » beaucoup moins courbées.

D. Fusarium roseum type $\mathbf{X}$ (Link) MESSIAEN et al. (1976), F. roseum (Link) Sn 2 H. var. gibbosum (Wollenw.) MESSIAEN \& CASSINI forme compactum

Non pathogène sur tubercules, ce champignon semble posséder les mêmes niches écologiques que les 2 champignons précédents ; sa fréquence élevée d'isolement nécessite une détermination correcte.

Bien que ce champignon soit extrêmement typique, il possède l'inconvénient d'avoir une dénomination indéterminée attribuée par MESSIAEN et al. (1976) qui le décrivent comme un type intermédiaire entre $F$. roseum var. graminearum et $F$. roseum var. culmorum.

La morphologie des colonies est proche de celle des 2 variétés précédentes avec, cependant, sur milieu TANAKA, le développement d'une zone centrale de mycélium aérien de couleur "vieux rose ", entourée d'une zone plus brune de couleur iodée. Sa vitesse de croissance varie entre 7,6 et $8 \mathrm{~mm}$ par jour.

Ses macroconidies évoquent celles de $F$. roseum var. graminearum : effilées, pointues à l'extrémité terminale, avec un pédicelle, souvent tronqué, à l'extrémité distale. Mais elles sont très nombreuses, toujours groupées en sporodochies de couleur marron foncé et possédant une longueur assez homogène (30-36 $\mu \mathrm{m})$. Ces macroconidies présentent un aspect dissymétrique : leur plus grande largeur $(5-6 \mu \mathrm{m})$ se situant dans le $1 / 3$ supérieur de la spore.

Ce champignon possède, par ailleurs, de très nombreuses chlamydospores intercalaires situées dans les hyphes (visibles après $8 \mathrm{j}$ de culture sur milieu TANAKA). Elles sont globuleuses, parfois uniques mais disposées souvent en chaînettes ou en groupes imbriqués de 2 ou 3. Leur double paroi de couleur iodée leur donne souvent l'aspect de ballon rigide.

Compte tenu de l'important taux d'isolement de $F$. roseum type $\mathrm{X}$, nous ne maintiendrons pas ce champignon sous cette appellation d'autant qu'il peut assez facilement être rattaché, en raison de ses nombreuses chlamydospores et de la forme de ses macroconidies à la variété gibbosum de MESSIAEN \& CAS SINI (1968). Ces auteurs regroupent, dans cette variété, de nombreuses espèces déterminées par WOLLENWEBER \& REINKING, et, plus particulièrement, $F$. compactum (Wollenw.) dont le type $\mathrm{X}$ semble se rapprocher en raison de sa pigmentation rouge, de sa sporulation abondante et de sa vitesse de croissance.

C'est pourquoi nous proposons de changer la déno- mination de $F$. roseum type $\mathrm{X}$ de MESSIAEN $e t$ al. (1976), en F. roseum (Link) Sn. \& H. var. gibbosum (Wollenw.) MESSIAEN \& CASSINI forme compactum (gibbosum en raison du nombre et du type des chlamydospores ainsi que de la forme des macroconidies ; compactum en raison de la pigmentation rouge, de la vitesse de croissance rapide et de la sporulation abondante).

\section{CONCLUSION \\ DISTRIBUTION ÉCOLOGIQUE DES DIFFÉRENTES ESPËCES OU VARIÉTÉS DE FUSARIUM}

Les différentes espèces ou variétés de Fusarium décrites précédemment constituent une flore très fluctuante qui évolue quantitativement et qualitativement lors des différentes étapes de la culture et de la conservation de la pomme de terre. Les travaux déjà réalisés permettent de situer avec précision la distribution de chacun des constituants de cette flore fusarienne (fig. 4).

\section{A. En conservation (TIVOLI \& JOUAN, 1981 ; TIVOLI \& BEDIN, 1982)}

Sur les tubercules en voie de pourriture, la flore est dominée par $F$. roseum var. sambucinum (qui représente environ 40 p. 100 des souches de Fusarium), puis par $F$. roseum var. arthrosporioides et $F$. solani var. coeruleum constituant respectivement 18 et 12 p. 100 des souches). Les autres variétés de $F$. roseum ne constituent chacune que 6 à 9 p. 100 des souches et $F$. oxysporum seulement 5 p. 100. L'importance relative du $1^{\text {er }}$ parasite s'explique fort bien en raison de ses capacités infectieuses importantes (TIVOLI et al., 1986a).

Dans les locaux de conservation, sur les murs, le sol ou le matériel, c'est $F$. solani var. coeruleum qui est le plus représenté puisqu'il constitue 56 p. 100 des souches. F. roseum var. sambucinum regroupe 20 p. 100 d'entre elles, tandis que les autres variétés de $F$. roseum ne représentent chacune que 5 à 7 p. 100 de la flore.

\section{B. En végétation (TIVOLI et al., 1986b)}

Sur les plantes, au niveau des différents organes (tiges, racines, stolons), c'est $F$. oxysporum qui prédomine puisqu'il représente respectivement, avant et après le défanage, de 89 à 67 p. 100 des souches isolées. Avant le défanage, les autres espèces ou variétés de Fusarium sont, soit très peu représentées (entre 2 et 4 p. 100 des souches pour les $F$. roseum, var. culmorum, var. sambucinum, var. arthrosporioides et var. gibbosum forme compactum), soit absentes (c'est le cas de $F$. roseum var. graminearum et $F$. solani var. coeruleum).

Après le défanage, l'ensemble des $F$. roseum augmente puisqu'il représente globalement 34 p. 100 des souches.

Dans le sol, $F$. solani var. coeruleum prédomine, il 


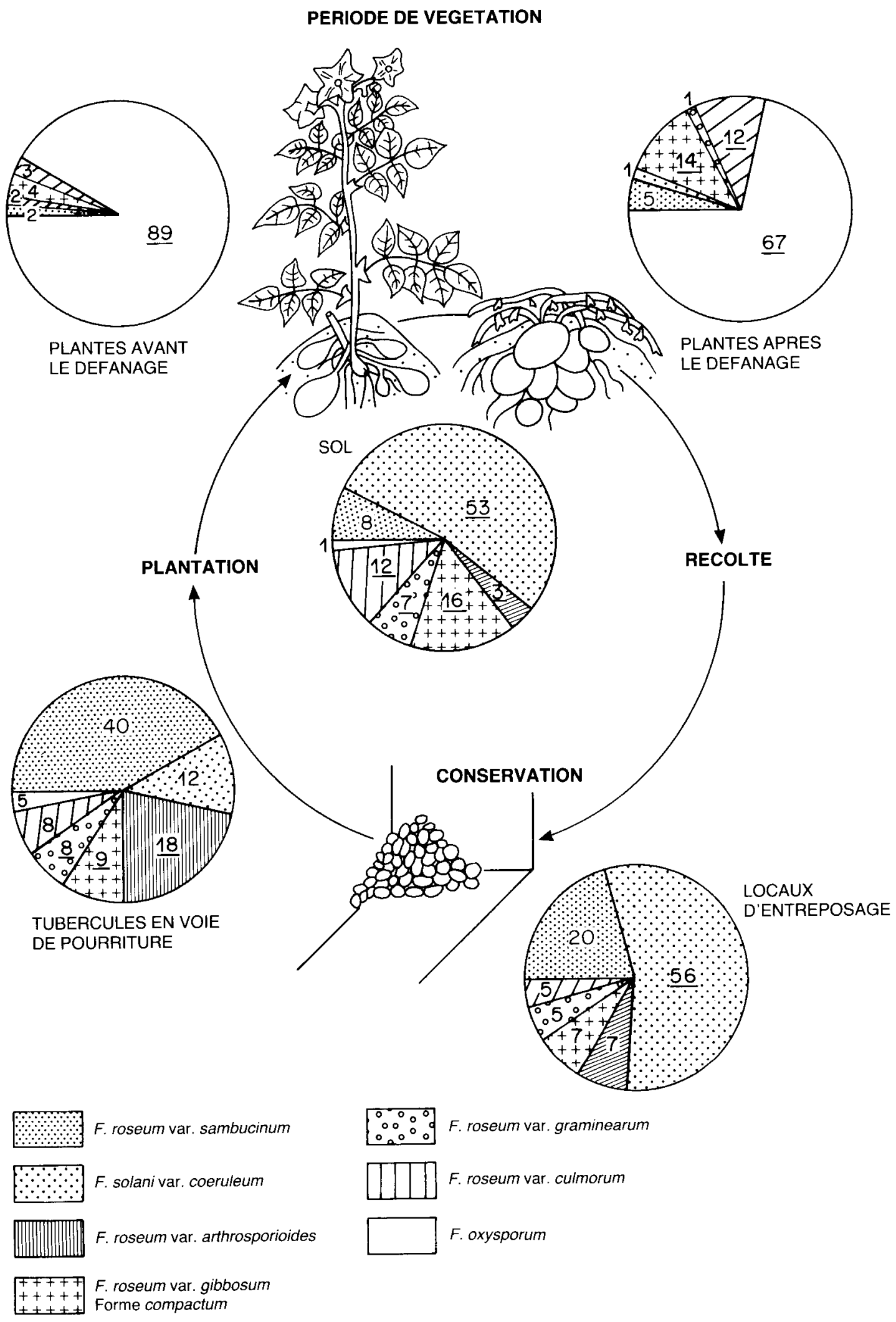

Figure 4

Fréquence des différentes espèces ou variétés de Fusarium rencontrées en France sur la pomme de terre et dans son environnement. (Exprimé en p. 100 de souches isolées.)

Frequency of different species and varieties of Fusarium found in France on potatoes and in their environment (\% of strains isolated). 
représente à lui seul 53 p. 100 des souches de Fusarium; les Fusarium roseum var. gibbosum forme compactum, culmorum et sambucinum représentent respectivement 16,12 et 8 p. 100 des souches. Il faut signaler, bien que $F$. oxysporum soit un hôte habituel du sol (MESSIAEN et al., 1968). qu'il n'est pas détecté par la méthode de piégeage sur les tranches de demitubercules.

\section{REMERCIEMENTS}

L'auteur tient à remercier Monsieur MESSIAEN (I.N.R.A., Montpellier), Messieurs MASSENOT et Molin (Revue Agronomie), ainsi que les Docteurs R. WASTIE (Scottish Crop Research Institute) et L. V. TuRkensteen (Pays-Bas) pour les conseils positifs qu'ils ont bien voulu apporter lors de la rédaction de cette publication.

Recu le 12 juillet 1987. Accepté le 4 janvier 1988.

\section{RÉFÉRENCES BIBLIOGRAPHIQUES}

Boissonnet-Menes M., Lecoq H., 1976. Transmission de virus par fusion de protoplastes chez Pyricularia oryzae. Physiol. vég., 14, 251-257.

Booth C., 1971. The genus Fusarium. C.M.I. Kew. Surrey, England, $237 \mathrm{p}$.

Booth C., 1977. Fusarium. Laboratory guide to the identification of the major species. C.M.I. Kew, Surrey, England, 58 p.

Cormack M. W., 1951. Variation in the cultural characteristics and pathogenicity of Fusarium avenaceum and $F$. arthrosporioides. Canad. J. Bot., 29, 32-45.

Gerlach W., Nirenberg H., 1982. The genus Fusarium, a pictorial atlas. Paul Parey, Berlin, 406 p.

Messiaen C. M., 1959. La systématique du genre Fusarium selon Snyder et Hansen. Rev. Pathol. vég. Entomol. agric. Fr., 38, 253266.

Messiaen C. M., Cassini R., 1968. Recherche sur les Fusarioses. IV - La systématique des Fusarium. Ann. Epiphyties, 19, 387-454.

Messiaen C. M., Belliard-Alonzo L., Barrière Y. de la Tullaye B., 1976. Etude qualitative des Fusarium roseum dans des sols des environs de Versailles, sous diverses rotations ou associations végétales. Ann. Phytopathol., 8, 269-281.

Nelson P. E., Toussoun T. A., Marasas W. F. O., 1983. Fusarium species. An illustrated manual for identification. Pennsylvania state University press, $193 \mathrm{p}$.
Ou S. H., 1972. Rice diseases. C.M.I. Kew, Surrey, England, 368 p.

Tivoli B., Jouan B., 1981. Inventaire, fréquence et agressivité des différentes espèces ou variétés de Fusarium responsables de la pourriture sèche des tubercules de pomme de terre. Agronomie, 1 (9), 787-794.

Tivoli B., Bedin P., 1982. Etude de la contamination fongique des locaux de conservation des pommes de terre de semence et possibilités de désinfection. Phytiatr. Phytopharm., 31, 61-70.

Tivoli B., Abdul Razzaq K., Jouan B., Lemarchand E., 1986a. Etude de la capacité infectieuse des espèces de Fusarium sur tubercules de pomme de terre. Potato Res., 29, 13-32.

Tivoli B., Jouan B., Lemarchand E., 1986b. Etude de la transmission des Fusarium par le tubercule de semence et par le sol. Potato Res., 29, 141-162.

Toussoun T. A., Nelson P. E., 1976. A pictorial guide to the identification of Fusarium species. Pennsylvania State University Press, $43 \mathrm{p}$.

Wollenweber H. W., Reinking O. A., 1935. Die Fusarien, ihre Beschreibung, Schadwirkung und Bekämpfung, Paul Parey, Berlin, $335 \mathrm{p}$. 